\title{
CURVATURE GROWTH OF SOME 4-DIMENSIONAL GRADIENT RICCI SOLITON SINGULARITY MODELS
}

\author{
BENNETT CHOW ${ }^{\mathrm{A}}$, MICHAEL FREEDMAN ${ }^{\mathrm{B}}$, HENRY SHIN $^{\mathrm{A}}$, \\ AND YONGJIA ZHANG ${ }^{\mathrm{A}}$
}

\begin{abstract}
In this note we discuss estimates for the curvature of 4dimensional gradient Ricci soliton singularity models by applying Perelman's point selection, a fundamental result of Cheeger and Naber, and topological lemmas.
\end{abstract}

\section{INTRODUCTION}

1.1. Definition of a singularity model. For a finite-time singular solution to the Ricci flow on a closed oriented manifold $\left(\mathcal{M}^{n}, g(t)\right), t \in[0, T)$, $T<\infty$, we have $\sup _{M \times[0, T)}|\mathrm{Rm}|=\infty$. An associated singularity model $\left(\mathcal{M}_{\infty}^{n}, g_{\infty}(t)\right), t \in(-\infty, 0]$, is a complete ancient solution which is a limit of pointed rescalings. More precisely, there exists a sequence of space-time points $\left(x_{i}, t_{i}\right)$ in $\mathcal{M} \times[0, T)$ with $K_{i} \doteqdot|\mathrm{Rm}|\left(x_{i}, t_{i}\right) \rightarrow \infty$ such that the sequence of pointed solutions $\left(\mathcal{M}, g_{i}(t),\left(x_{i}, 0\right)\right)$, where $g_{i}(t)=K_{i} g\left(K_{i}^{-1} t+t_{i}\right)$ and $t \in\left[-K_{i} t_{i}, 0\right]$, converges in the $C^{\infty}$ pointed Cheeger-Gromov sense to the complete ancient solution $\left(\mathcal{M}_{\infty}, g_{\infty}(t),\left(x_{\infty}, 0\right)\right), t \in(-\infty, 0]$, for some $x_{\infty} \in \mathcal{M}_{\infty}$. Note that $g_{\infty}(t)$ is not assumed to have bounded curvature on each time slice.

A folklore conjecture is that any singularity model must have bounded curvature. In dimension 3, this is true by the work of Perelman [47]. Observe that it is not obvious that singularity models are necessarily of finite (topological) type. Neither is it obvious that singularity models are embeddable in the compact manifold from which they arise. However, if a singularity model has finite type, then it is embeddable in the original compact manifold and thus has an orientation induced by the embedding.

1.2. Classification of 3-dimensional singularity models. There is now a complete classification of 3-dimensional singularity models. We shall use GRS as an abbreviation for gradient Ricci soliton. Firstly, 3-dimensional noncollapsed shrinking GRS with bounded curvature have been classified by Hamilton [26] and by Perelman [48], who proved nonexistence in the noncompact positive sectional curvature case. Naber [45] showed that shrinking

Key words and phrases. Ricci flow, gradient Ricci soliton, singularity model, Ricci flat, asymptotically locally Euclidean manifold. 
GRS with bounded curvature must be noncollapsed. It was shown that 3dimensional shrinking GRS must have bounded curvature via the works of Cao, Chen, and Zhu [12] and Ni and Wallach [46], with related works by Chen [16] and Petersen and Wylie [49]. Secondly, Brendle [8] proved the assertion by Perelman that the only 3-dimensional nonflat noncollapsed steady GRS is the rotationally symmetric Bryant soliton. Thirdly, the works of Hamilton [26], Perelman [47], and Brendle [10], prove that any 3-dimensional singularity model must be either a shrinking GRS or a steady GRS. In particular, Brendle [10] proved Perelman's conjecture that any 3dimensional noncollapsed ancient solution with positive bounded sectional curvature must be a steady GRS. See Bamler and Kleiner [5] for a later, alternative treatment related to their work on strong stability of 3-dimensional Ricci flow and their proof of the generalized Smale conjecture [3, 4]. Finally, one obtains from Hamilton, Perelman, and Brendle's results that the possible 3-dimensional oriented singularity models are classified as: a shrinking spherical space form $S^{3} / \Gamma$, a round cylinder $S^{2} \times \mathbb{R}$ or its $\mathbb{Z}_{2}$-quotient, or the Bryant soliton.

There has been much progress on the understanding of higher-dimensional shrinking GRS, largely due to the works of Munteanu and Wang (see e.g. $[43,44]$ and the references therein), with the strongest results in dimension 4. Wylie [51] proved that the fundamental group is finite. Kotschwar and Wang proved an important uniqueness result [31]; see also [30, 32]. Haslhofer and Müller $[27,28]$ have proved precompactness theorems. Y. Li and B. Wang [36] proved an improved no local collapsing theorem for shrinking GRS.

For recent progress on steady GRS, see Appleton [1], Brendle [9], Cao and Cui [13], Deng and Zhu [21, 22, 23], Deruelle [24], Munteanu and Sesum [38], and Munteanu, Sung, and Wang [39], Munteanu and Wang [40, 41, 42], and the references therein. For the classification of 4-dimensional shrinking GRS with positive isotropic curvature, see Li, Ni, and Wang [35]. Very recently, Chen, Deruelle, and Sun [20] proved that any 4-real-dimensional Kähler shrinking GRS with scalar curvature tending to zero at infinity must be the Feldman-Ilmanen-Knopf shrinker [25].

In the case where the potential function is constant, a steady GRS is a Ricci-flat manifold. Assuming also that this manifold is a singularity model, by Perelman's no local collapsing theorem it is necessarily $\kappa$-noncollapsed on all scales. In particular, it has Euclidean volume growth. In dimension 4, by a fundamental result of Cheeger and Naber [15], it must be a Ricci-flat asymptotically locally Euclidean (ALE) manifold. We remark that singularity models of 4-dimensional singular solutions with bounded scalar curvature have been shown to be such ALE manifolds by Bamler and Zhang [6] and Simon [50].

1.3. Main results. In this paper we study the curvature growth of 4dimensional GRS singularity models. 
Theorem 1. Any 4-dimensional steady GRS singularity model $(\mathcal{M}, g, f)$ must have bounded curvature, that is, there exists a constant $C$ depending on the $G R S$ such that $|\mathrm{Rm}| \leq C$ on $\mathcal{M}$.

For similar reasons, we have:

Theorem 2. Any 4-dimensional shrinking GRS singularity model must have curvature which grows at most quadratically. That is, for any o $\in \mathcal{M}$ there exists a constant $C$ depending only on the GRS and o such that $|\mathrm{Rm}|(x) \leq$ $C(d(x, o)+1)^{2}$ for all $x \in \mathcal{M}$.

The paper is organized as follows. In Section 2 we first recall Perelman's point selection method on Riemannian manifolds, which is generally used to obtain limits. We then discuss under what conditions the local derivative of curvature estimates of Shi yield instantaneous estimates for GRS. Next, we prove the main Theorems 1 and 2 modulo the topological lemmas proved in Section 3.

For a survey of 4-dimensional Ricci flow, see [18] by Gompf, Hillman, and two of the authors.

\section{Curvature estimates for GRS singularity models}

2.1. Point selection. The following is Perelman's point selection method; see [47]. Since the method is crucial to our results, we include its proof for the sake of completeness.

Lemma 3. Let $\left(\mathcal{M}^{n}, g\right)$ be a complete Riemannian manifold. For any $y_{0} \in$ $\mathcal{M}$ (let $\left.P_{0} \doteqdot|\mathrm{Rm}|\left(y_{0}\right)\right)$ and $A_{0} \in \mathbb{R}^{+}$there exists $x_{0} \in B_{2 A_{0} P_{0}^{-1 / 2}}\left(y_{0}\right)$ such that $Q_{0} \doteqdot|\mathrm{Rm}|\left(x_{0}\right) \geq P_{0}$ and

$$
|\mathrm{Rm}| \leq 4 Q_{0} \quad \text { in } B_{A_{0} Q_{0}^{-1 / 2}}\left(x_{0}\right) .
$$

For example, we may choose $A_{0}=\frac{1}{3} P_{0}^{1 / 2}$, in which case we also have $A_{0} Q_{0}^{-1 / 2} \leq \frac{1}{3}$, so that $x_{0} \in B_{2 / 3}\left(y_{0}\right)$ and $B_{A_{0} Q_{0}^{-1 / 2}}\left(x_{0}\right) \subset B_{1}\left(y_{0}\right)$.

Proof. If (1) holds for $x_{0}=y_{0}$, then we are done. So suppose (1) does not hold for $x_{0}=y_{0}$. Let $O_{0} \doteqdot|\operatorname{Rm}|\left(y_{0}\right)=P_{0}$. Since (1) does not hold for $x_{0}=y_{0}$, there exists $y_{1} \in B_{A_{0} O_{0}^{-1 / 2}}\left(y_{0}\right)$ such that

$$
O_{1} \doteqdot|\operatorname{Rm}|\left(y_{1}\right)>4 O_{0}
$$

By induction, suppose that $y_{0}, \ldots, y_{j}$ have been chosen such that (1) does not hold for $x_{0}=y_{k}$ for all $0 \leq k \leq j-1$ and with $O_{k} \doteqdot|\operatorname{Rm}|\left(y_{k}\right)$ for $0 \leq k \leq j$ we have $y_{k+1} \in B_{A_{0} O_{k}^{-1 / 2}}\left(y_{k}\right)$ satisfies $O_{k+1}>4 O_{k}$ for $0 \leq k \leq j-1$. Then, for $0 \leq k \leq j$ we have

$$
O_{k} \geq 4^{k} O_{0}=4^{k} P_{0}
$$


and

$$
\begin{aligned}
d\left(y_{k}, y_{0}\right) & =\sum_{\ell=0}^{k-1} d\left(y_{\ell+1}, y_{\ell}\right) \\
& <\sum_{\ell=0}^{k-1} A_{0} O_{\ell}^{-1 / 2} \\
& \leq A_{0} \sum_{\ell=0}^{k-1} O_{0}^{-1 / 2} 2^{-\ell} \\
& <2 A_{0} O_{0}^{-1 / 2} .
\end{aligned}
$$

Hence $y_{k} \subset B_{2 A_{0} O_{k}^{-1 / 2}}\left(y_{0}\right)$ and $B_{A_{0} O_{k}^{-1 / 2}}\left(y_{k}\right) \subset B_{3 A_{0} O_{k}^{-1 / 2}}\left(y_{0}\right)$ for $0 \leq k \leq j$.

For the sequence $y_{0}, y_{1}, \ldots$ there exists a first $j_{0}$ for which (1) holds for $x_{0}=y_{j_{0}}$, for otherwise we would have an infinite sequence of points $\left\{y_{k}\right\}_{k=0}^{\infty}$ for which $|\mathrm{Rm}|\left(y_{k}\right) \geq 4^{k} P_{0}$ and $y_{k} \in B_{2 A_{0} O_{0}^{-1 / 2}}\left(y_{0}\right)$ for all $k \geq 0$, a contradiction. The lemma follows from taking $x_{0}=y_{j_{0}}$ since $|\mathrm{Rm}|\left(y_{j_{0}}\right) \geq 4^{j_{0}} P_{0} \geq P_{0}$ and since (3) implies $d\left(y_{j_{0}}, y_{0}\right)<2 A_{0} P_{0}^{-1 / 2}$.

To summarize, given any $A_{0}>0$ and point $y_{0}$, Perelman's point selection method finds a nearby point $x_{0}$ such that $|\mathrm{Rm}|$ in the ball centered at $x_{0}$ of scaled radius $A_{0}$ is bounded by 4 times its value at $x_{0}$. This is effective since $A_{0}$ is arbitrary. So, given a sequence $\left\{y_{i}\right\}$ with $|\operatorname{Rm}|\left(y_{i}\right) \rightarrow \infty$, we may choose $\left\{A_{i}\right\}$ so that $A_{i} \rightarrow \infty$. In particular, as an immediate consequence of the lemma, we have:

Proposition 4. Let $\left(\mathcal{M}^{n}, g\right)$ be a complete Riemannian manifold. For any sequence $\left\{y_{i}\right\}_{i=1}^{\infty}$ in $\mathcal{M}$ with $P_{i} \doteqdot|\mathrm{Rm}|\left(y_{i}\right) \rightarrow \infty$ there exists a sequence $\left\{x_{i}\right\}_{i=1}^{\infty}$ in $\mathcal{M}$ such that for each $i \geq 1$ we have $x_{i} \in B_{2 / 3}\left(y_{i}\right)$, $Q_{i} \doteqdot|\mathrm{Rm}|\left(x_{i}\right) \geq P_{i}$, and

$$
|\mathrm{Rm}| \leq 4 Q_{i} \quad \text { in } B_{A_{i} Q_{i}^{-1 / 2}}\left(x_{i}\right), \quad \text { where } A_{i}=\frac{1}{3} P_{i}^{1 / 2} \rightarrow \infty .
$$

2.2. Instantaneous local derivative estimates. We have the following (instantaneous in time) local derivative estimates. This is useful for rescalings about points where $|\mathrm{Rm}|$ is bounded below by a positive constant.

Lemma 5. Let $\left(\mathcal{M}^{n}, g, f\right)$ be a complete steady or shrinking GRS. Suppose that $p \in \mathcal{M}, r>0$, and $C$ are such that in $B_{2 r}(p)$ we have $|\mathrm{Rm}| \leq C r^{-2}$ and $|\nabla f| \leq r^{-1}$. Then $\left|\nabla^{m} \mathrm{Rm}\right| \leq C_{m} r^{-2-m}$ in $B_{r}(p)$, where $C_{m}$ depends only on $m, C$, and $n$.

Proof. Let $(\mathcal{M}, g(t), f(t)), \lambda t<1$, be the canonical form associated to the steady GRS; see e.g. Theorem 4.1 in [19]. Here $\lambda=0$ in the steady case and $\lambda=1$ in the shrinking case, so that $\lambda \geq 0$. By hypothesis, $|\operatorname{Rm}|(x, 0) \leq$ 
$C r^{-2}$ for $x \in B_{2 r}^{g(0)}(p)$. By the definition of the canonical form,

$$
\begin{array}{r}
\frac{\partial}{\partial t} \varphi_{t}(x)=\frac{1}{1-\lambda t}\left(\nabla_{g(0)} f(0)\right)\left(\varphi_{t}(x)\right), \\
g(t)=(1-\lambda t) g(0), \text { and } f(t)=f(0) \circ \varphi_{t} \text { satisfy } \\
\frac{\partial}{\partial t} g(t)=-2 \mathrm{Rc}=2 \nabla_{g(t)}^{2} f(t)-\frac{\lambda}{1-\lambda t} g(t) .
\end{array}
$$

That is, $(\mathcal{M}, g(t))$ is a solution to the Ricci flow evolving by scaling and by the pullback by a 1-parameter family of diffeomorphisms. Hence, by the assumption that $|\nabla f(0)|_{g(0)} \leq r^{-1}$ in $B_{2 r}^{g(0)}(p)$, we have the inequality

$$
\left|\frac{\partial}{\partial t} \varphi_{t}(x)\right|_{g(0)} \leq r^{-1}
$$

whenever $\varphi_{t}(x) \in B_{2 r}^{g(0)}(p)$ and $t \leq 0$. Hence, if $x \in B_{3 r / 2}^{g(0)}(p)$ and $t \in\left[-\frac{r^{2}}{2}, 0\right]$, then

$$
d_{g(0)}\left(\varphi_{t}(x), x\right) \leq \int_{t}^{0}\left|\frac{\partial}{\partial t} \varphi_{\bar{t}}(x)\right|_{g(0)} d \bar{t} \leq \frac{r}{2} .
$$

Thus $\varphi_{t}(x) \in B_{2 r}^{g(0)}(p)$, so that $|\operatorname{Rm}|(x, t)=|\operatorname{Rm}|\left(\varphi_{t}(x), 0\right) \leq C r^{-2}$. By Shi's local derivative of curvature estimates, $\left|\nabla^{m} \mathrm{Rm}\right|(x, 0) \leq C_{m} r^{-2-m}$ for $x \in B_{r}^{g(0)}(p)$.

The condition $|\nabla f| \leq r^{-1}$ puts some restriction on the scales $r$ for which we may apply this lemma. For instance, on a steady GRS it is only known that $|\nabla f|^{2}=1-R \leq 1$; hence this results works naturally for $0<r \leq 1$ (or less than any fixed constant). On the other hand, on a shrinking GRS, since we only have $|\nabla f| \leq \sqrt{f-R} \leq \frac{1}{2} d(o, x)+C$, this lemma works naturally for $0<r \lesssim d^{-1}(o, x)$. In particular, for the steady case, we have:

Corollary 6. Let $\left(\mathcal{M}^{n}, g, f\right)$ be a complete steady $G R S$ with $|\mathrm{Rm}| \leq C r^{-2}$ in $B_{2 r}(p)$, where $r \in(0,1]$. Then $\left|\nabla^{m} \mathrm{Rm}\right| \leq C_{m} r^{-2-m}$ in $B_{r}(p)$, where $C_{m}$ depends only on $m, C$, and $n$.

2.3. Four-dimensional Ricci-flat ALE manifolds. We say that a complete noncompact oriented Riemannian 4-manifold $\left(\mathcal{M}^{4}, g\right)$ is asymptotically locally Euclidean $(A L E)$ if there exists $\tau>0$, a compact subset $K$, a finite subgroup $\Gamma$ of $S O(4)$ acting freely on $S^{3}$, and an orientation-preserving diffeomorphism $\Phi:\left(\mathbb{R}^{4}-B_{C}(0)\right) / \Gamma \rightarrow \mathcal{M}-K$ for some $C$ such that the pullback $\tilde{h}$ to the cover $\mathbb{R}^{4}-B_{C}(0)$ of $h=\Phi^{*} g$ satisfies $\partial_{I}\left(\tilde{h}_{j k}-\delta_{j k}\right)=O\left(|x|^{-\tau-|I|}\right)$ for each multi-index $I=\left(i_{1}, i_{2}, i_{3}, i_{4}\right)$. By Bando, Kasue, and Nakajima [7, Theorem 1.5], if an ALE manifold is Ricci flat, then there exists $\Phi$ with $\tau=4$.

It is conjectured that any simply-connected Ricci-flat ALE 4-manifold must be hyperkähler, the latter of which has been classified by Kronheimer 
$[33,34]$. For progress in this direction, see Lock and Viaclovsky [37]. Appleton [2] has shown that the Eguchi-Hanson space, which is a hyperkähler ALE, can occur as a singularity model of 4-dimensional Ricci flow.

2.4. Steady GRS singularity models. Our first main result is the following.

Proposition 7. If $\left(\mathcal{M}^{4}, g, f\right)$ is a steady GRS which is also a singularity model, then $|\mathrm{Rm}|$ is bounded.

Proof. Suppose $|\mathrm{Rm}|$ is not bounded. Then there exists $\left\{y_{i}\right\}_{i=1}^{\infty}$ in $\mathcal{M}$ with $P_{i} \doteqdot|\operatorname{Rm}|\left(y_{i}\right) \rightarrow \infty$. By Proposition 4 , there exists a sequence $\left\{x_{i}\right\}_{i=1}^{\infty}$ in $\mathcal{M}$ such that for each $i \geq 1$ we have $x_{i} \in B_{2 / 3}\left(y_{i}\right), Q_{i} \doteqdot|\mathrm{Rm}|\left(x_{i}\right) \geq P_{i} \geq 100$, and

(4) $\quad|\mathrm{Rm}| \leq 4 Q_{i} \quad$ in $B_{A_{i} Q_{i}^{-1 / 2}}\left(x_{i}\right)$, where $A_{i}=\frac{1}{3} P_{i}^{1 / 2} \rightarrow \infty$.

Since $\left(\mathcal{M}^{4}, g, f\right)$ is a singularity model, it is $\kappa$-noncollapsed on all scales for some $\kappa>0$. Hence (4) implies

$$
\operatorname{Vol} B_{Q_{i}^{-1 / 2}}\left(x_{i}\right) \geq \kappa Q_{i}^{-2} \text { for all } i \geq 1 .
$$

Let $\left(\mathcal{M}^{4}, g(t), f(t)\right), t \in(-\infty, \infty)$, be the associated canonical form, where $g(t)=\varphi_{t}^{*} g$ and $f(t)=f \circ \varphi_{t}$. Since $\frac{\partial}{\partial t} \varphi_{t}(x)=\left(\nabla_{g(0)} f(0)\right)\left(\varphi_{t}(x)\right)$, we have $\left|\frac{\partial}{\partial t} \varphi_{t}(x)\right|_{g(0)} \leq 1$. Hence, if $x \in B_{\left(A_{i}-1\right) Q_{i}^{-1 / 2}}^{g(0)}\left(x_{i}\right)$ and $t \in\left[-Q_{i}^{-1}, 0\right]$, then

$$
d_{g(0)}\left(\varphi_{t}(x), x\right) \leq|t| \leq Q_{i}^{-1} \leq Q_{i}^{-1 / 2},
$$

so that $\varphi_{t}(x) \in B_{A_{i} Q_{i}^{-1 / 2}}^{g(0)}\left(x_{i}\right)$ and hence $|\operatorname{Rm}|(x, t)=|\operatorname{Rm}|\left(\varphi_{t}(x), 0\right) \leq 4 Q_{i}$. Hence, by Shi's local derivative estimates, we have

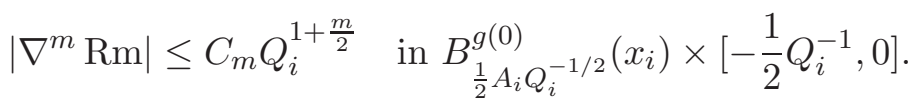

By the above and by Hamilton's Cheeger-Gromov compactness theorem, there exists a subsequence such that $\left(\mathcal{M}^{4}, Q_{i} g\left(Q_{i}^{-1} t\right), x_{i}\right)$ converges in the $C^{\infty}$ pointed Cheeger-Gromov sense to a complete solution to the Ricci flow $\left(\mathcal{M}_{\infty}^{4}, g_{\infty}(t), x_{\infty}\right), t \in\left[-\frac{1}{2}, 0\right]$, with $\left|\operatorname{Rm}_{g_{\infty}}\right| \leq 4$ and $\left|\operatorname{Rm}_{g_{\infty}}\right|\left(x_{\infty}, 0\right)=1$. In particular, $g_{\infty}(t)$ is nonflat. Since $\left(\mathcal{M}^{4}, g, f\right)$ is a steady GRS, we have $0 \leq R \leq 1$. This and $Q_{i} \rightarrow \infty$ imply that $R_{g_{\infty}} \equiv 0$, which in turn implies that $\operatorname{Rc}_{g_{\infty}} \equiv 0$ by the Ricci flow equation $\frac{\partial R_{g_{\infty}}}{\partial t}=\Delta_{g_{\infty}} R_{g_{\infty}}+2\left|\mathrm{Rc}_{g_{\infty}}\right|^{2}$.

By Perelman's no local collapsing theorem and since $R_{g_{\infty}(0)}=0$ and we are on a singularity model (a limit of a limit is a limit), there exists $\kappa>0$ such that $\operatorname{Vol}_{g_{\infty}(0)} B_{r}^{g_{\infty}(0)}\left(x_{\infty}\right) \geq \kappa r^{4}$ for all $r>0$ (see e.g. Theorems 28.6 and 28.9 in [17]) $;^{1}$ hence by definition $g_{\infty}(0)$ has Euclidean volume growth.

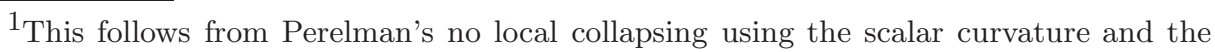
fact that the rescaling factors tend to infinity.
} 
By Cheeger and Naber's theorem (Corollary 8.86 of [15]), $\left(\mathcal{M}_{\infty}, g_{\infty}(0)\right)$ is asymptotically locally Euclidean (ALE).

Since $\left(\mathcal{M}_{\infty}^{4}, g_{\infty}(0)\right)$ is a nonflat Ricci-flat ALE 4-manifold, there exists a finite subgroup $\Gamma$ of $\mathrm{SO}(4)$ such that the end of $\mathcal{M}_{\infty}$ is diffeomorphic to $\left(\mathcal{S}^{3} / \Gamma\right) \times(0,1){ }^{2}$ Let $\mathcal{S}^{3} / \Gamma \subset \mathcal{M}_{\infty}$ be embedded so that the noncompact region in $\mathcal{M}_{\infty}$ bounded by $\mathcal{S}^{3} / \Gamma$ is diffeomorphic to $\left(\mathcal{S}^{3} / \Gamma\right) \times[0,1)$. Let $\Omega$ denote the compact region in $\mathcal{M}_{\infty}$ bounded by $\mathcal{S}^{3} / \Gamma$. Then $\Omega^{\circ}$ is diffeomorphic to $\mathcal{M}_{\infty}$. By the definition of Cheeger-Gromov convergence, there exist an exhaustion $U_{i}$ of $\mathcal{M}_{\infty}$ and embeddings $\psi_{i}: U_{i} \rightarrow B_{\frac{1}{2} A_{i} Q_{i}^{-1 / 2}}^{g(0)}\left(x_{i}\right)$. In particular, $\psi_{i}(\Omega)$ are embeddings of $\Omega$ with $\psi_{i}(\Omega) \subset B_{1}^{g(0)}\left(x_{i}\right)$ for each $i$ sufficiently large. So we may assume that the $\psi_{i}(\Omega)$ are disjoint from each other in the steady GRS $\mathcal{M}$.

By hypothesis, there exists a solution to the Ricci flow on a closed 4manifold $\left(\mathcal{N}^{4}, h(t)\right), t \in[0, T), T<\infty$, for which the steady GRS $(\mathcal{M}, g, f)$ is a singularity model. Hence there exist an exhaustion $\left\{V_{j}\right\}$ of $\mathcal{M}$ and embeddings $\phi_{j}: V_{j} \rightarrow \mathcal{N}$. In particular, for any $I \in \mathbb{N}$ there exists $j(I)$ such that $V_{j(I)}$ contains $\psi_{i}(\Omega)$ for all $1 \leq i \leq I$. Then the $\Omega_{i} \doteqdot\left(\phi_{j(I)} \circ \psi_{i}\right)(\Omega)$ are disjoint embeddings into $\mathcal{N}$ for $1 \leq i \leq I$. Since the $\Omega_{i}^{\circ}$ are each diffeomorphic to $\mathcal{M}_{\infty}$ and are pairwise disjoint and since $I$ is arbitrary, we obtain a contradiction to Theorem 12 below.

2.5. Shrinking GRS singularity models. By a similar argument we can prove the following.

Proposition 8. If $\left(\mathcal{M}^{4}, g, f\right)$ is a shrinking GRS which is also a singularity model and $o \in \mathcal{M}$, then there exists a constant $C$ such that

$$
|\mathrm{Rm}|(x) \leq C(d(x, o)+1)^{2} \quad \text { for all } x \in \mathcal{M}
$$

Proof. Suppose there exists a sequence of points in the shrinking GRS $\left\{y_{i}\right\}_{i=1}^{\infty}$ in $\mathcal{M}$ with $P_{i}\left(d\left(y_{i}, o\right)+1\right)^{-2} \rightarrow \infty$, where $P_{i} \doteqdot|\operatorname{Rm}|\left(y_{i}\right)$. By a previous proposition, there exists a sequence $\left\{x_{i}\right\}_{i=1}^{\infty}$ in $\mathcal{M}$ such that for each $i \geq 1$ we have $x_{i} \in B_{2 / 3}\left(y_{i}\right), Q_{i} \doteqdot|\mathrm{Rm}|\left(x_{i}\right) \geq P_{i}$, and

$$
|\mathrm{Rm}| \leq 4 Q_{i} \quad \text { in } B_{A_{i} Q_{i}^{-1 / 2}}\left(x_{i}\right), \quad \text { where } A_{i}=\frac{1}{3} P_{i}^{1 / 2} \rightarrow \infty
$$

Let $\left(\mathcal{M}^{4}, g(t), f(t)\right), t \in(-\infty, 1)$, be the associated canonical form, where $g(t)=(1-t) \varphi_{t}^{*} g$ and $f(t)=f \circ \varphi_{t}$. Since $\frac{\partial}{\partial t} \varphi_{t}(x)=\frac{1}{1-t}\left(\nabla_{g(0)} f(0)\right)\left(\varphi_{t}(x)\right)$,

\footnotetext{
${ }^{2}$ A nonflat Ricci-flat ALE 4-manifold has only one end since otherwise it will split as the product of a line and a Ricci flat three-manifold, which in turn implies that it is flat.
} 
we have

$$
\begin{aligned}
\left|\frac{\partial}{\partial t} \varphi_{t}(x)\right|_{g(0)} & \leq \frac{1}{1-t}\left|\nabla_{g(0)} f(0)\right|\left(\varphi_{t}(x)\right) \\
& \leq \frac{1}{1-t} f^{1 / 2}\left(\varphi_{t}(x), 0\right) \\
& \leq \frac{1}{1-t}\left(d_{g(0)}\left(\varphi_{t}(x), o\right)+C\right) \\
& \leq d_{g(0)}\left(\varphi_{t}(x), o\right)+C
\end{aligned}
$$

for $t \leq 0$ since $\left|\nabla\left(f^{1 / 2}(0)\right)\right|_{g(0)} \leq 1$ by $R \geq 0$. Hence, if $x \in B_{\frac{3}{4} A_{i} Q_{i}^{-1 / 2}}^{g(0)}\left(x_{i}\right)$ and $t \in\left[-Q_{i}^{-1}, 0\right]$, then

$$
\begin{aligned}
d_{g(0)}\left(\varphi_{t}(x), x\right) & \leq \int_{t}^{0}\left|\frac{\partial}{\partial t} \varphi_{t}(x)\right|_{g(0)} d t \\
& \leq \int_{t}^{0}\left(d_{g(0)}\left(\varphi_{t}(x), o\right)+C\right) d t \\
& \leq C Q_{i}^{-1}\left(d_{g(0)}\left(x_{i}, o\right)+C\right) \\
& \ll Q_{i}^{-1 / 2}
\end{aligned}
$$

since $Q_{i}\left(d_{g(0)}\left(x_{i}, o\right)+1\right)^{-2} \gtrsim P_{i}\left(d_{g(0)}\left(y_{i}, o\right)+1\right)^{-2} \rightarrow \infty$. Thus $\varphi_{t}(x) \in$ $B_{A_{i} Q_{i}^{-1 / 2}}^{g(0)}\left(x_{i}\right)$ and hence $|\mathrm{Rm}|(x, t)=|\operatorname{Rm}|\left(\varphi_{t}(x), 0\right) \leq 4 Q_{i}$. Hence we may apply Shi's local derivative estimates and Hamilton's Cheeger-Gromov compactness theorem to obtain that there exists a subsequence such that $\left(\mathcal{M}^{4}, Q_{i} g\left(Q_{i}^{-1} t\right), x_{i}\right)$ converges in the $C^{\infty}$ pointed Cheeger-Gromov sense to a complete solution to the Ricci flow $\left(\mathcal{M}_{\infty}^{4}, g_{\infty}(t), x_{\infty}\right), t \in\left[-\frac{1}{2}, 0\right]$, with $\left|\mathrm{Rm}_{g_{\infty}}\right| \leq 4$ and $\left|\operatorname{Rm}_{g_{\infty}}\right|\left(x_{\infty}, 0\right)=1$. Since $\left(\mathcal{M}^{4}, g, f\right)$ is a shrinking GRS, we have $0<R \leq f$. This and $Q_{i} f^{-1}\left(x_{i}\right) \rightarrow \infty$ imply that $R_{g_{\infty}} \equiv 0$, which in turn implies that $\mathrm{Rc}_{g_{\infty}} \equiv 0$ and so $g_{\infty}(0)$ is ALE. The remainder of the proof will be the same as in the steady GRS case.

\subsection{Steady GRS models with curvature not decaying.}

Proposition 9. Let $\left(\mathcal{M}^{4}, g, f\right)$ be a steady GRS which is also a singularity model. If there exist a constant $c>0$ and a sequence $y_{i} \rightarrow \infty$ such that

$$
|\operatorname{Rm}|\left(y_{i}\right) \geq c \text { for all } i,
$$

then $\left(\mathcal{M}, g, y_{i}\right)$ subconverges to a non-Ricci-flat steady GRS with bounded curvature.

Proof. Observe that we may assume that $\left(\mathcal{M}^{4}, g\right)$ is not Ricci flat, since otherwise there does not exist a sequence $\left\{y_{i}\right\}$ as in the hypothesis by Cheeger and Naber's aforementioned theorem. Since the singularity model has bounded curvature by Proposition 7, it follows from Shi's local derivative 
estimates (or Lemma 5) that the covariant derivative of curvature of each order is uniformly bounded. Consider the sequence $\left\{\left(\mathcal{M}, g, f_{i}, y_{i}\right)\right\}_{i=1}^{\infty}$, where $f_{i}(x)=f(x)-f\left(y_{i}\right)$. We have $\left|\nabla f_{i}\right|=|\nabla f|=\sqrt{1-R}$ and $\left|\nabla^{2} f\right|=\mid$ Rc $\mid$ are both uniformly bounded. By applying Shi's Bernstein-type estimates and by the covariant derivatives of curvature bounds, it is not hard to show that the covariant derivatives of $f_{i}$ of each order are bounded independent of $i$. Taking into account the noncollapsing condition, we may extract a subsequence from $\left\{\left(\mathcal{M}, g, f_{i}, y_{i}\right)\right\}_{i=1}^{\infty}$ which converges in the pointed smooth CheegerGromov sense to $\left(\mathcal{M}_{\infty}^{4}, g_{\infty}, f_{\infty}, y_{\infty}\right)$. It then follows from the smooth convergence that

$$
\begin{aligned}
\mathrm{Rc}_{\infty}+\nabla^{2} f_{\infty} & =0, \\
\left|\nabla f_{\infty}\right|^{2}+R_{\infty} & =1 .
\end{aligned}
$$

The limit is evidently a steady GRS. It is also nonflat since

$$
c \leq|\operatorname{Rm}|\left(y_{i}\right) \rightarrow\left|\operatorname{Rm}_{\infty}\right|\left(y_{\infty}\right) \geq c>0 .
$$

To see that it is not Ricci flat, we assume for a contradiction that $g_{\infty}$ is Ricci flat. Then immediately we have

$$
\begin{aligned}
\nabla^{2} f_{\infty} & =0 \\
\left|\nabla f_{\infty}\right|^{2} & =1 .
\end{aligned}
$$

Hence $\left(\mathcal{M}_{\infty}, g_{\infty}\right)$ splits as the product of a line and a three-dimensional Ricci-flat manifold, which is flat; a contradiction.

2.7. Shrinking GRS models with quadratic curvature growth. The following result is proved using similar methods.

Proposition 10. Let $\left(\mathcal{M}^{4}, g, f\right)$ be a shrinking GRS which is also a singularity model and let $o \in \mathcal{M}$. If there exist a constant $c>0$ and a sequence $y_{i} \rightarrow \infty$ such that

$$
|\operatorname{Rm}|\left(y_{i}\right) \geq c\left(d\left(y_{i}, o\right)+1\right)^{2} \quad \text { for all } i,
$$

then there exists $x_{i} \rightarrow \infty$ with associated rescalings limiting to a non-Ricciflat steady GRS with bounded curvature.

Proof. Let $\left\{x_{i}\right\}, Q_{i}$, and $A_{i}$ be as in Proposition 4. By (5), we have that if $x \in B_{\frac{3}{4} A_{i} Q_{i}^{-1 / 2}}^{g(0)}\left(x_{i}\right)$ and $t \in\left[-Q_{i}^{-1}, 0\right]$, then

$$
d_{g(0)}\left(\varphi_{t}(x), x\right) \leq C Q_{i}^{-1}\left(d_{g(0)}\left(x_{i}, o\right)+C\right) \leq C Q_{i}^{-1 / 2} .
$$

Since $A_{i} \rightarrow \infty$, we have $\varphi_{t}(x) \in B_{A_{i} Q_{i}^{-1 / 2}}^{g\left(x_{i}\right)}$ for $i$ sufficiently large. As in the proof of Proposition 8, there exists a subsequence such that $\left(\mathcal{M}, Q_{i} g\left(Q_{i}^{-1} t\right), x_{i}\right)$ converges in the $C^{\infty}$ pointed Cheeger-Gromov sense to a complete solution to the Ricci flow $\left(\mathcal{M}_{\infty}^{4}, g_{\infty}(t), x_{\infty}\right), t \in\left[-\frac{1}{2}, 0\right]$, with 
$\left|\mathrm{Rm}_{g_{\infty}}\right| \leq 4$ and $\left|\mathrm{Rm}_{g_{\infty}}\right|\left(x_{\infty}, 0\right)=1$. Let $g_{i}=Q_{i} g$ and let $f_{i}(x)=$ $f(x)-f\left(x_{i}\right)$. We have

$$
\mathrm{Rc}_{g_{i}}+\nabla_{g_{i}}^{2} f_{i}=\frac{1}{2} Q_{i}^{-1} g_{i} .
$$

For any $A>0$ and for any $x \in B_{A}^{g_{i}}\left(x_{i}\right)$, we have

$$
\left|\nabla f_{i}\right|_{g_{i}}^{2}+R_{g_{i}}=\frac{f}{Q_{i}} \geq \frac{\inf \left\{f(y): y \in B^{g} Q_{i}^{-\frac{1}{2}}\left(x_{i}\right)\right\}}{C\left(d_{g}\left(x_{i}, o\right)+1\right)^{2}} \geq c>0,
$$

for all $i$ large enough, where $c>0$ is independent of $A$ and where we have used the lower bound for the potential function of Cao and Zhou [14]. Furthermore, we have

$$
\begin{gathered}
\left|\nabla f_{i}\right|_{g_{i}}(x)=Q_{i}^{-1 / 2}|\nabla f|(x) \leq Q_{i}^{-1 / 2} f^{1 / 2}(x) \leq C, \\
\left|\nabla_{g_{i}}^{2} f_{i}\right|_{g_{i}}=\left|\operatorname{Rc}_{g_{i}}-\frac{1}{2} Q_{i}^{-1} g_{i}\right|_{g_{i}} \leq C,
\end{gathered}
$$

and

$$
\left|\nabla_{g_{i}}^{k} f_{i}\right|_{g_{i}}=\left|\nabla_{g_{i}}^{k-2} \mathrm{Rc}_{g_{i}}\right|_{g_{i}} \leq C_{k} \quad \text { for } k \geq 3 .
$$

Hence, by taking $i \rightarrow \infty$ we obtain $f_{i} \rightarrow f_{\infty}$ in $C^{\infty}$ with respect to the Cheeger-Gromov convergence of $g_{i}$ to $g_{\infty}=g_{\infty}(0)$, where

$$
\begin{aligned}
\left|\nabla_{g_{\infty}} f_{\infty}\right|_{g_{\infty}}^{2}+R_{g_{\infty}} & =c_{0}>0 \\
\operatorname{Rc}_{g_{\infty}}+\nabla_{g_{\infty}}^{2} f_{\infty} & =0 .
\end{aligned}
$$

Notice that the left-hand-side of the first equation is constant because the steady GRS equation holds by the second equation; it follows from (6) that this constant is positive. According to our point selection procedure, the limit steady GRS $\left(\mathcal{M}_{\infty}^{4}, g_{\infty}, f_{\infty}\right)$ is nonflat and has bounded curvature. To see that it is non-Ricci-flat, we assume the contrary and obtain

$$
\begin{aligned}
\left|\nabla_{g_{\infty}} f_{\infty}\right|_{g_{\infty}}^{2} & =c_{0}>0, \\
\nabla_{g_{\infty}}^{2} f_{\infty} & =0 .
\end{aligned}
$$

It follows immediately that $\left(\mathcal{M}_{\infty}, g_{\infty}\right)$ splits as the product of a line and a three-dimensional Ricci-flat manifold and hence $g_{\infty}$ must be flat; this is a contradiction.

One would like to show that steady GRS limits as in the proposition above are not possible, in which case it would follow for a 4-dimensional shrinking GRS singularity model that $|\mathrm{Rm}|=\mathrm{o}\left(d^{2}\right)$ (this would imply finite topological type).

Remark 11. For the exclusion of Ricci-flat ALE limits in the proofs of Propositions 9 and 10, we may alternatively use Theorem 12 below. 


\section{EXCluding AN unbounded NUMBER OF COPIES OF A RICCI-FLAT}

ALE 4-MANIFOLD

3.1. Statement of the result. In this section we prove the result which we applied in the previous section to exclude there existing an unbounded number of disjoint copies of a Ricci-flat ALE 4-manifold in a closed 4-manifold.

Theorem 12. If $\mathcal{N}$ is a closed 4-manifold and if $\mathcal{A}$ is a Ricci-flat $A L E$ 4-manifold, then there can exist at most a bounded number of disjoint copies of $\mathcal{A}$ embedded in $\mathcal{N}$.

3.2. Spherical space form ends of 4-manifolds having an unbounded number of disjoint embeddings. We first prove the following. ${ }^{3}$

Proposition 13. Let $\mathcal{M}_{0}$ be a noncompact 4-manifold without boundary and with a single end diffeomorphic to $S^{3} / \Gamma \times[0, \infty)$, where $S^{3} / \Gamma$ is a spherical space form. If there exists a compact 4 -manifold $\mathcal{N}$ containing an unbounded number of disjoint copies of $\mathcal{M}_{0}$, then $H_{1}\left(S^{3} / \Gamma, \mathbb{Z}\right)$ is a direct double, i.e., isomorphic to $A \oplus A$ for some abelian group $A$.

Let $\mathcal{M}_{0}$ be as in the hypotheses of the proposition. Add $S^{3} / \Gamma$ to $\mathcal{M}_{0}$ as its boundary to obtain a compact manifold $\mathcal{M}$ with boundary $\partial \mathcal{M}=S^{3} / \Gamma \doteqdot \partial$; so, $\mathcal{M}_{0}$ is the interior of $\mathcal{M}$.

Lemma 14. Under the hypotheses of Proposition 13, the homomorphism $H_{m}(\partial ; R) \rightarrow H_{m}(\mathcal{M} ; R)$ induced by $\partial \hookrightarrow \mathcal{M}$ is onto for $m=0,1,2,3$ and any coefficient ring $R$. The cases $R \cong \mathbb{Z}$ and $R \cong \mathbb{Z}_{p}$ will be useful for us. We suppress writing the coefficients, $R$, in the proof.

Proof. This is clear for $m=0$. Applying Mayer-Vietoris to one copy of $\mathcal{M}$ and to the complement of its interior $\overline{\mathcal{N}}-\mathcal{M}$, which have intersection $\partial$, we obtain the following exact sequence:

$\cdots \rightarrow H_{m}(\partial) \stackrel{\left(i_{*}, j_{*}\right)}{\longrightarrow} H_{m}(\mathcal{M}) \oplus H_{m}(\overline{\mathcal{N}-\mathcal{M}}) \stackrel{k_{*}-l_{*}}{\longrightarrow} H_{m}(\mathcal{N}) \stackrel{\partial_{*}}{\longrightarrow} H_{m-1}(\partial) \rightarrow \cdots$,

where $i: \partial \hookrightarrow \mathcal{M}, j: \partial \hookrightarrow \overline{\mathcal{N}-\mathcal{M}}, k: \mathcal{M} \hookrightarrow \mathcal{N}$, and $l: \overline{\mathcal{N}-\mathcal{M}} \hookrightarrow \mathcal{N}$ are the inclusion maps. Suppose that $i_{*}: H_{m}(\partial) \rightarrow H_{m}(\mathcal{M})$ is not onto. Then there exists a class $\alpha \in H_{m}(\mathcal{M})$ which is not in the image of $i_{*}$. Thus, for any such $\alpha$ and any $\beta \in H_{m}(\overline{\mathcal{N}-\mathcal{M}})$, we have $k_{*}(\alpha)-l_{*}(\beta) \neq 0$.

Thus the cokernel of $i_{*}, \operatorname{coker}\left(i_{*}\right)=H_{m}(\mathcal{M}) / i_{*}\left(H_{m}(\partial)\right)$, injects into $H_{m}(\mathcal{N}) / l_{*}\left(H_{m}(\overline{\mathcal{N}-\mathcal{M}})\right.$ by the map induced by $k_{*}$. Indeed, firstly observe that this map is well defined since $k_{*}\left(i_{*}\left(H_{m}(\partial)\right)\right)=l_{*}\left(j_{*}\left(H_{m}(\partial)\right) \subset\right.$ $l_{*}\left(H_{m}(\overline{\mathcal{N}-\mathcal{M}})\right.$. Secondly, suppose that there is a nonzero coset $\alpha i_{*}\left(H_{m}(\partial)\right)$ which maps to zero. Then we have that $\alpha \in H_{m}(\mathcal{M})$ is not in the image of $i_{*}$ and that $k_{*}(\alpha) \in l_{*}\left(H_{m}(\overline{\mathcal{N}-\mathcal{M}})\right)$, which is a contradiction.

Now suppose that $\mathcal{N}$ contains a finite sequence of disjoint embedded copies of $\mathcal{M}$, denoted by $\left\{\mathcal{M}_{p}\right\}_{p=1}^{I}$, for $I$ arbitrarily large. Let $\mathcal{M}^{I}=\bigcup_{p=1}^{I} \mathcal{M}_{p}$

\footnotetext{
${ }^{3}$ One of the many references for the standard results from algebraic topology used in this section is Hatcher's book [29].
} 
and $\partial^{I}=\partial \mathcal{M}^{I}=\cup_{p=1}^{I} \partial \mathcal{M}_{p}$. Let $i^{I}: \partial^{I} \hookrightarrow \mathcal{M}^{I}$ and $l^{I}: \overline{\mathcal{N}-\mathcal{M}^{I}} \hookrightarrow \mathcal{N}$ denote the inclusion maps. Since, in the argument above we may replace $\mathcal{M}$ by $\mathcal{M}^{I}$ (we did not assume connectedness), we have that

$$
\operatorname{coker}\left(i_{*}^{I}\right)=H_{m}\left(\mathcal{M}^{I}\right) / i_{*}^{I}\left(H_{m}\left(\partial^{I}\right)\right)=\oplus_{p=1}^{I} \operatorname{coker}\left(i_{p *}\right)
$$

injects into $H_{m}(\mathcal{N}) / l_{*}^{I}\left(H_{m}\left(\overline{\mathcal{N}-\mathcal{M}^{I}}\right)\right.$, where $i_{p}: \partial \mathcal{M}_{p} \hookrightarrow \mathcal{M}_{p}$ is the inclusion map. In this way, we obtain an injection from modules of increasing rank into $H_{m}(\mathcal{N}) / l_{*}^{I}\left(H_{m}\left(\overline{\mathcal{N}-\mathcal{M}^{I}}\right)\right.$. This contradicts the finite dimensionality of $H_{m}(\mathcal{N})$.

Remark 15. One may easily picture the workings of the preceding formal argument: If there is any class $\alpha$ (of any dimension $m$ ) in a copy of $\mathcal{M}$ in $\mathcal{N}$ which does not come from the boundary $\partial$, it is effectively 'locked into' that copy of $\mathcal{M}$; such classes can neither die in $\mathcal{N}$ nor participate in relations among themselves, because intersecting such null-homologies or homologies (respectively) with $\partial$ would yield a cycle in $\partial$ mapping onto $\alpha$, contrary to assumption. In other words, the intuition is that the cokernel in each copy of $\mathcal{M}$ is isolated like an island and cannot have any relation with other classes.

By Lemma 14, the long exact sequence of the pair $(\mathcal{M}, \partial)$ yields the three short exact sequences. Setting $R \cong \mathbb{Z}$, we have:

$$
\begin{aligned}
& 0 \rightarrow H_{2}(\mathcal{M}, \partial ; \mathbb{Z}) \rightarrow H_{1}(\partial ; \mathbb{Z}) \rightarrow H_{1}(\mathcal{M} ; \mathbb{Z}) \rightarrow 0 \\
& 0 \rightarrow H_{3}(\mathcal{M}, \partial ; \mathbb{Z}) \rightarrow H_{2}(\partial ; \mathbb{Z}) \rightarrow H_{2}(\mathcal{M} ; \mathbb{Z}) \rightarrow 0 \\
& 0 \rightarrow H_{4}(\mathcal{M}, \partial ; \mathbb{Z}) \rightarrow H_{3}(\partial ; \mathbb{Z}) \rightarrow H_{3}(\mathcal{M} ; \mathbb{Z}) \rightarrow 0
\end{aligned}
$$

Since $\partial$ is a spherical space form, $H_{1}(\partial ; \mathbb{Z})$ is torsion, and by $(7)$ so is $H_{1}(\mathcal{M} ; \mathbb{Z})$. By Poincaré duality and the cohomology universal coefficient theorem (see [29, Theorem 3.2 and Corollary 3.3]), we have that $H_{2}(\partial ; \mathbb{Z}) \cong$ $H^{1}(\partial ; \mathbb{Z}) \cong \mathbb{Z}^{b_{1}(\partial)} \oplus$ Tor $H_{0}(\partial, \mathbb{Z}) \cong 0$, where $b_{1}(\partial)$ is the first Betti number of $\partial$. Thus (8) implies

$$
H_{2}(\mathcal{M} ; \mathbb{Z}) \cong 0
$$

We also have that $H_{3}(\partial ; \mathbb{Z}) \cong \mathbb{Z}$, but maps to zero in $H_{3}(\mathcal{M} ; \mathbb{Z})$ by $(9)$ since $H_{4}(\mathcal{M}, \partial ; \mathbb{Z}) \rightarrow H_{3}(\partial ; \mathbb{Z})$ is an isomorphism. Thus $H_{3}(\mathcal{M} ; \mathbb{Z}) \cong 0$.

By Lemma 14 with $R=\mathbb{Z}_{p}$, that is, by (7) with coefficients $\mathbb{Z}_{p}$ instead of $\mathbb{Z}$, we have

$$
0 \rightarrow H_{2}\left(\mathcal{M}, \partial ; \mathbb{Z}_{p}\right) \rightarrow H_{1}\left(\partial ; \mathbb{Z}_{p}\right) \rightarrow H_{1}\left(\mathcal{M} ; \mathbb{Z}_{p}\right) \rightarrow 0
$$

By Lefschetz duality (see $[29$, Theorem 3.43$]), H_{2}\left(\mathcal{M}, \partial ; \mathbb{Z}_{p}\right) \cong H^{2}\left(\mathcal{M} ; \mathbb{Z}_{p}\right)$. By the cohomology universal coefficient theorem,

$0 \rightarrow \operatorname{Ext}_{\mathbb{Z}}^{1}\left(H_{1}(\mathcal{M} ; \mathbb{Z}), \mathbb{Z}_{p}\right) \rightarrow H^{2}\left(\mathcal{M} ; \mathbb{Z}_{p}\right) \rightarrow \operatorname{Hom}_{\mathbb{Z}}\left(H_{2}(\mathcal{M} ; \mathbb{Z}), \mathbb{Z}_{p}\right) \cong 0 \rightarrow 0$

As observed below $(9), H_{1}(\mathcal{M} ; \mathbb{Z})$ is torsion; thus

$$
H_{1}\left(\mathcal{M} ; \mathbb{Z}_{p}\right) \cong \operatorname{Hom}_{\mathbb{Z}}\left(\operatorname{Tor}\left(H_{1}(\mathcal{M} ; \mathbb{Z})\right), \mathbb{Z}_{p}\right)
$$


But this agrees with the usual ${ }^{4}$ formula which calculates $\left.\operatorname{Ext}_{\mathbb{Z}}^{1} H_{1}(\mathcal{M} ; \mathbb{Z}), \mathbb{Z}_{p}\right)$. Putting this together with (12) yields:

$$
H^{2}\left(\mathcal{M} ; \mathbb{Z}_{p}\right) \cong \operatorname{Ext}_{\mathbb{Z}}^{1}\left(H_{1}(\mathcal{M} ; \mathbb{Z}), \mathbb{Z}_{p}\right) \cong H_{1}\left(\mathcal{M} ; \mathbb{Z}_{p}\right) .
$$

These observations applied to (11) yield:

$$
H_{1}\left(\partial ; \mathbb{Z}_{p}\right)=H_{1}\left(\mathcal{M} ; \mathbb{Z}_{p}\right) \oplus H_{1}\left(\mathcal{M} ; \mathbb{Z}_{p}\right)
$$

To bring this information back towards integer coefficients, apply the homology universal coefficient theorem, for $X=\mathcal{M}$ and $\partial$ :

$$
0 \rightarrow H_{1}(X ; \mathbb{Z}) \otimes \mathbb{Z}_{p} \rightarrow H_{1}\left(X ; \mathbb{Z}_{p}\right) \rightarrow \operatorname{Tor}\left(H_{0}(X ; \mathbb{Z}), \mathbb{Z}_{p}\right) \cong 0 \rightarrow 0
$$

to get

$$
H_{1}(\partial ; \mathbb{Z}) \otimes \mathbb{Z}_{p} \cong H_{1}(\mathcal{M} ; \mathbb{Z}) \otimes \mathbb{Z}_{p} \oplus H_{1}(\mathcal{M} ; \mathbb{Z}) \otimes \mathbb{Z}_{p} \quad \text { for all primes } p .
$$

This still does not quite prove Proposition 13. We need to exclude possibilities like $H_{1}(\partial ; \mathbb{Z}) \cong \mathbb{Z}_{4} \oplus \mathbb{Z}_{2}$ and $H_{1}(\mathcal{M} ; \mathbb{Z}) \cong \mathbb{Z}_{2}$. This can be done by returning to line $(7)$ : order $H_{1}(\partial ; \mathbb{Z})=\left(\operatorname{order} H_{2}(\mathcal{M}, \partial ; \mathbb{Z})\right) \times\left(\operatorname{order} H_{1}(\mathcal{M} ; \mathbb{Z})\right)$, but $H_{2}(\mathcal{M}, \partial ; \mathbb{Z}) \cong H^{2}(\mathcal{M} ; \mathbb{Z}) \cong \operatorname{Ext}_{\mathbb{Z}}^{1}\left(H_{1}(\mathcal{M} ; \mathbb{Z}), \mathbb{Z}\right) \cong$ torsion $H_{1}(\mathcal{M} ; \mathbb{Z}) \cong$ $H_{1}(\mathcal{M} ; \mathbb{Z})$, so order $H_{1}(\partial ; \mathbb{Z})=\left(\text { order } H_{1}(\mathcal{M} ; \mathbb{Z})\right)^{2}$. Proposition 13 follows from this, (14), and the fundamental theorem of finite abelian groups.

3.3. Proof of Theorem 12. We may now prove Theorem 12 using Bamler's argument in $\S 6$ of [52]. For a contradiction, suppose that there exists a nonflat Ricci-flat ALE 4-manifold $\mathcal{A}$ that admits unbounded collections of disjoint embeddings in a compact 4-manifold $\mathcal{N}$. By Proposition 13, the spherical space form $S^{3} / \Gamma, \Gamma$ nontrivial, of the end of $\mathcal{A}$ satisfies $H_{1}\left(S^{3} / \Gamma, \mathbb{Z}\right) \cong A \oplus A$ for some abelian group $A$. This implies (see e.g. Lemma 6.3 in [52]) that either $\Gamma$ is the binary dihedral group $D_{n}^{*}$ with $n$ even or $\Gamma$ is the binary icosahedral group of order 120 . Now, by Lemma 6.5 in $[52], b_{2}(\mathcal{A}) \geq 1$, which contradicts $(10)$.

Remark 16. We may also rule out the case where $\Gamma$ is the binary icosahedral group $I^{*}$ of order 120 , that is, where $\partial=S^{3} / I^{*}$ is the Poincaré homology sphere, as follows. By Rochlin's theorem, if $\partial \mathcal{M}=S^{3} / I^{*}$, then $H_{*}(\mathcal{M} ; \mathbb{Z}) \approx$ $H_{*}(\mathrm{pt} ; \mathbb{Z})$. As in Lemma 14 , the facts that $H_{*}\left(S^{3} / I^{*} ; \mathbb{Z}\right) \cong H_{*}\left(S^{3} ; \mathbb{Z}\right)$ and $H_{*}(\mathcal{M} ; \mathbb{Z}) \nRightarrow H_{*}(\mathrm{pt} ; \mathbb{Z})$ imply, via Mayer-Vietoris, that $H_{*}(\mathcal{N} ; \mathbb{Z})$ is infinitely generated, a contradiction.

Acknowledgment. We would like to thank the referee for a number of helpful suggestions.

\footnotetext{
${ }^{4}$ See for example the computations on the Wikipedia page Ext functor or Hatcher's book [29]. According to these formulae, $\operatorname{Ext}_{\mathbb{Z}}^{1}\left(\mathbb{Z}, \mathbb{Z}_{p}\right) \cong 0$ and $\operatorname{Ext}_{\mathbb{Z}}^{1}\left(\right.$ torsion, $\left.\mathbb{Z}_{\mathrm{p}}\right) \cong$ $\operatorname{Hom}_{\mathbb{Z}}\left(\right.$ torsion, $\left.\mathbb{Z}_{\mathrm{p}}\right)$. We use these formulae and the fact that Ext commutes with direct sums.
} 


\section{REFERENCES}

[1] Appleton, Alexander. A family of non-collapsed steady Ricci solitons in even dimensions greater or equal to four. arXiv:1708.00161.

[2] Appleton, Alexander. Eguchi-Hanson singularities in U(2)-invariant Ricci flow. arXiv:1903.09936.

[3] Bamler, Richard H.; Kleiner, Bruce. Uniqueness and stability of Ricci flow through singularities. arXiv:1709.04122.

[4] Bamler, Richard H.; Kleiner, Bruce. Ricci flow and diffeomorphism groups of 3manifolds. arXiv:1712.06197.

[5] Bamler, Richard H.; Kleiner, Bruce. On the rotational symmetry of 3-dimensional $\kappa$-solutions. arXiv:1904.05388.

[6] Bamler, Richard H.; Zhang, Qi S. Heat kernel and curvature bounds in Ricci flows with bounded scalar curvature. Adv. Math. 319 (2017), 396-450.

[7] Bando, Shigetoshi; Kasue, Atsushi; Nakajima, Hiraku. On a construction of coordinates at infinity on manifolds with fast curvature decay and maximal volume growth. Invent. Math. 97 (1989), no. 2, 313-349.

[8] Brendle, Simon. Rotational symmetry of self-similar solutions to the Ricci flow. Inventiones Math. 194 (2013), 731-764.

[9] Brendle, Simon. Rotational symmetry of Ricci solitons in higher dimensions. J. Differential Geom. 97 (2014), 191-214.

[10] Brendle, Simon. Ancient solutions to the Ricci flow in dimension 3. arXiv:1811.02559v2.

[11] Chan, Pak-Yeung. Curvature estimates for steady Ricci solitons. Trans. Amer. Math. Soc. 372 (2019), 8985-9008.

[12] Cao, Huai-Dong; Chen, Bing-Long; Zhu, Xi-Ping. Recent developments on Hamilton's Ricci flow. Surveys in differential geometry. Vol. XII. Geometric flows, 47-112, Int. Press, Somerville, MA, 2008.

[13] Cao, Huai-Dong; Cui, Xin. Curvature estimates for four-dimensional gradient steady Ricci solitons. J. Geom. Anal. 30 (2020), 511--525.

[14] Cao, Huai-Dong; Zhou, De-Tang. On complete gradient shrinking Ricci solitons. J. Differential Geom. 85 (2010), 175-186.

[15] Cheeger, Jeff; Naber, Aaron. Regularity of Einstein manifolds and the codimension 4 conjecture. Annals of Mathematics 182 (2015), 1093-1165.

[16] Chen, Bing-Long. Strong uniqueness of the Ricci flow. J. Differential Geom. 82 (2009), 363-382.

[17] Chow, Bennett; Chu, Sun-Chin; Glickenstein, David; Guenther, Christine; Isenberg, Jim; Ivey, Tom; Knopf, Dan; Lu, Peng; Luo, Feng; Ni, Lei. The Ricci flow: techniques and applications. Part IV: long-time solutions and related topics. Mathematical Surveys and Monographs, 206, AMS, Providence, RI, 2015.

[18] Chow, Bennett; Freedman, Michael; Gompf, Robert; Hillman, Jonathan. Survey of Ricci flow and 4-manifolds. In preparation.

[19] Chow, Bennett; Lu, Peng; Ni, Lei. Hamilton's Ricci flow. Lectures in Contemporary Mathematics, 3, Science Press and Graduate Studies in Mathematics, 77, American Mathematical Society (co-publication), 2006.

[20] Conlon, Ronan J.; Deruelle, Alix; Sun, Song. Classification results for expanding and shrinking gradient Kähler-Ricci solitons. arXiv:1904.0014.

[21] Deng, Yuxing; Zhu, Xiaohua Asymptotic behavior of positively curved steady Ricci solitons. Trans. Amer. Math. Soc. 370 (2018), no. 4, 2855--2877.

[22] Deng, Yuxing; Zhu, Xiaohua. Three-dimensional steady gradient Ricci solitons with linear curvature decay. Int. Math. Res. Not. IMRN 2019 (2019), no. 4, 1108-1124.

[23] Deng, Yuxing; Zhu, Xiaohua. Classification of gradient steady Ricci solitons with linear curvature decay. Sci. China Math. 63 (2020), 135-154. 
[24] Deruelle, Alix Steady gradient Ricci soliton with curvature in $L^{1}$. Comm. Anal. Geom. 20 (2012), no. 1, 31--53.

[25] Feldman, Mikhail; Ilmanen, Tom; Knopf, Dan. Rotationally symmetric shrinking and expanding gradient Kähler-Ricci solitons. J. Differential Geom. 65 (2003), 169-209.

[26] Hamilton, Richard S. The formation of singularities in the Ricci flow. Surveys in differential geometry, Vol. II (Cambridge, MA, 1993), 7-136, Internat. Press, Cambridge, MA, 1995.

[27] Haslhofer, Robert; Müller, Reto. A compactness theorem for complete Ricci shrinkers. Geom. Funct. Anal. 21 (2011), 1091-1116.

[28] Haslhofer, Robert; Müller, Reto. A note on the compactness theorem for 4d Ricci shrinkers. Proc. Amer. Math. Soc. 143 (2015), 4433-4437.

[29] Hatcher, Allen. Algebraic topology. Cambridge University Press, Cambridge, 2002.

[30] Kotschwar, Brett Kählerity of shrinking gradient Ricci solitons asymptotic to Kähler cones. J. Geom. Anal. 28 (2018), no. 3, 2609--2623.

[31] Kotschwar, Brett; Wang, Lu. Rigidity of asymptotically conical shrinking gradient Ricci solitons. J. Differential Geom. 100 (2015), 55-108.

[32] Kotschwar, Brett; Wang, Lu. Isometries of asymptotically conical shrinking Ricci solitons. arXiv:1901.00044.

[33] Kronheimer, Peter B. The construction of ALE spaces as hyper-Kähler quotients. J. Differential Geom. 29 (1989), no. 3, 665-683.

[34] Kronheimer, Peter B. A Torelli-type theorem for gravitational instantons. J. Differential Geom. 29 (1989), no. 3, 685-697.

[35] Li, Xiaolong; Ni, Lei; Wang, Kui. Four-dimensional gradient shrinking solitons with positive isotropic curvature. Int. Math. Res. Not. IMRN 2018 (2018), no. 3, 949 - 959.

[36] Li, Yu; Wang, Bing. Heat kernel on Ricci shrinkers. arXiv:1901.05691.

[37] Lock, Michael T.; Viaclovsky, Jeff A. Quotient singularities, eta invariants, and selfdual metrics. Geom. Topol. 20 (2016), 1773-1806.

[38] Munteanu, Ovidiu; Sesum, Natasa. On gradient Ricci solitons. J. Geom. Anal. 23 (2013), no. 2, 539-561.

[39] Munteanu, Ovidiu; Sung, Chiung-Jue; Wang, Jiaping. Poisson equation on complete manifolds. Adv. Math. 348 (2019), 81-145.

[40] Munteanu, Ovidiu; Wang, Jiaping. Smooth metric measure spaces with non-negative curvature. Comm. Anal. Geom. 19 (2011), no. 3, 451-486.

[41] Munteanu, Ovidiu; Wang, Jiaping. Analysis of weighted Laplacian and applications to Ricci solitons. Comm. Anal. Geom. 20 (2012), no. 1, 55-94.

[42] Munteanu, Ovidiu; Wang, Jiaping. Geometry of manifolds with densities. Adv. Math. 259 (2014), 269-305.

[43] Munteanu, Ovidiu; Wang, Jiaping. Geometry of shrinking Ricci solitons. Compositio Mathematica 151 (2015), 2273-2300.

[44] Munteanu, Ovidiu; Wang, Jiaping. Conical structure for shrinking Ricci solitons. J. Eur. Math. Soc. 19 (2017), 3377-3390.

[45] Naber, Aaron. Noncompact shrinking four solitons with nonnegative curvature. Journal für die Reine und Angewandte Mathematik 645 (2010), 125-153.

[46] Ni, Lei; Wallach, Nolan. On a classification of gradient shrinking solitons. Math. Res. Lett. 15 (2008), 941-955.

[47] Perelman, Grisha. The entropy formula for the Ricci flow and its geometric applications. arXiv:math.DG/0211159.

[48] Perelman, Grisha. Ricci flow with surgery on three-manifolds. arXiv:math.DG/ 0303109.

[49] Petersen, Peter; Wylie, William. On the classification of gradient Ricci solitons. Geom. Topol. 14 (2010), 2277-2300

[50] Simon, Miles. Extending four dimensional Ricci flows with bounded scalar curvature. arXiv:1504.02623. 
[51] Wylie, William. Complete shrinking Ricci solitons have finite fundamental group. Proc. Amer. Math. Soc. 136 (2008), 1803-1806.

[52] Zhang, Yongia. Compactness theorems for 4-dimensional gradient Ricci solitons. Pacific J. Math. 303 (2019), No. 1, 361-384.

A Department of Mathematics, University of California, San Diego, California 92093, $U S A$.

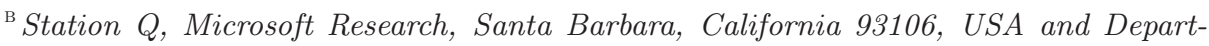
ment of Mathematics, University of California, Santa Barbara, California 93106, USA. 